\title{
AIAA 2003-3312
}

Large-Eddy Simulation of a Mach

0.75 Jet

Niklas Andersson*, Lars-Erik Eriksson*广 and Lars

Davidson*

* Department of Thermo and Fluid Dynamics, Chalmers University of Technology, SE-412 96 Göteborg, Sweden

$\dagger$ Volvo Aero Corporation, Military Engines Division, SE-461 81 Trollhättan, Sweden

\section{9th AIAA/CEAS Aeroacoustic Conference May 12-14, 2003/Hilton Head, South Carolina}




\title{
Large-Eddy Simulation of a Mach 0.75 Jet
}

\author{
Niklas Andersson*, Lars-Erik Eriksson*† and Lars Davidson* \\ * Department of Thermo and Fluid Dynamics, Chalmers \\ University of Technology, SE-412 96 Göteborg, Sweden \\ † Volvo Aero Corporation, Military Engines Division, \\ SE-461 81 Trollhättan, Sweden
}

\begin{abstract}
A large-eddy simulation (LES) of a compressible nozzle/jet configuration has been carried out. A cold Mach 0.75 jet was simulated. The Reynolds number based on the jet velocity at the nozzle exit plane and the nozzle diameter was 50 000. The Favre filtered Navier-Stokes equations were solved using a finite volume method with a low dissipative third order upwind scheme for the convective fluxes, a second order centered difference approach for the viscous fluxes and a three-stage second order Runge-Kutta time marching technique. A compressible form of Smagorinsky's sub-grid scale model was used for computation of the sub-grid scale stresses. The calculations were performed using a block structured boundary fitted mesh with approximately 3000000 cells. The calculations have been performed on a parallel computer, using message-passing interface (MPI). Absorbing boundary conditions based on characteristic variables were adopted for all free boundaries. Velocity components specified at the entrainment boundaries were estimated from a corresponding Reynolds Averaged Navier-Stokes (RANS) calculation. In order to diminish disturbances caused by the outlet boundary a buffer layer was added at the domain outlet. Kirchhoff surface integration has been utilized to obtain far-field sound pressure levels in a number of observer locations using instantaneous pressure from the LES. Aerodynamic results and predicted sound pressure levels are both in good agreement with experiments. Experimental data were provided by Laboratoire d'Etude Aeródynamiques, Poitiers. ${ }^{1-3}$
\end{abstract}

\section{Nomenclature} Published by the American Institute of Aeronautics and Astronautics, Inc. with permission.

$\begin{array}{ll}\mathcal{T} & \text { integral time scale } \\ L_{c} & \text { potential core length } \\ \tau_{r} & \text { retarded time } \\ \mathbf{x} & \text { observer location } \\ \mathbf{y} & \text { Kirchoff surface location } \\ \langle\ldots\rangle_{t} & \text { time averaged quantity } \\ \langle\ldots\rangle_{\theta} & \text { circumferentially averaged quantity }\end{array}$

\section{Subscripts}

$\begin{array}{ll}\infty & \text { free stream or ambient conditions } \\ j & \text { jet, nozzle exit condition } \\ 0 & \text { total condition } \\ c & \text { center line }\end{array}$

Superscripts

c convection

SGS sub-grid scale

$\sim \quad$ Favre filtered quantity

- $\quad$ spatially filtered quantity

\section{Introduction}

Restrictions of noise levels in the surroundings of airports have made reduction of near ground operation noise an important issue for aircraft and engine manufacturers. Noise generation has lately become a serious concern early in the design process. At take off the main sources of noise are the 
propelling jet and the engine fan. An EU project, $\mathrm{JEAN}^{1}$, with focus to investigate jet noise both numerically and experimentally and with a long term aim to improve existing noise predicting tools, was started in February 2001. The work to be presented has been performed within this project.

Numerical methods based on computational fluid dynamics (CFD) used for prediction of flow induced sound are often referred to as computational aeroacoustics (CAA). Using a grid fine enough in the far-field regions to not introduce sound propagation errors, the sound pressure level can be obtained directly from the flow field simulation. This requires a detailed numerical compressible flow simulation, e.g. direct numerical simulation (DNS), see for example Freund ${ }^{4}$ and Mitchell et al., ${ }^{5}$ or large-eddy simulation (LES), as done by for example Bogey et al. ${ }^{6}$ and Mankbadi et al. ${ }^{7}$

To save computational time, a hybrid approach may be used in which the computational problem is divided into two parts. A LES or DNS can be used to obtain the non-linear near-field, which in the jet noise case corresponds to the hydrodynamic jet region. Sources of sound obtained from the flow simulation are then propagated to far-field observer locations using either a solver based on the Linearized Euler Equations (LEE), see Billson et al., ${ }^{8}$ or an integral method such as Lighthill's acoustic analogy, as done by Bogey et al., ${ }^{9}$ or Kirchhoff surface integration, see Freund et al. ${ }^{10}$ In DNS all scales of the turbulent flow field are computed accurately, which requires a mesh fine enough to capture the smallest scales in the flow, whereas in LES the large scales of the flow are resolved and the influence on the large scales of the smaller, not resolved scales, are modelled using a sub-grid model. With the computational resources available today, DNS is restricted to fairly simple geometries and low Reynolds number flows. Moreover, it is believed, see Mankbadi, ${ }^{11}$ that large scales are more efficient than smaller ones in generating sound, which justifies the use of LES for sound predictions.

Another approach is to use a less computationally expensive RANS calculation to obtain a time averaged flow field. Information about length and time scales in the time averaged flow field can then be used to synthesize turbulence in the noise source regions, see Billson. ${ }^{12}$ This method is promising since simulations of high Reynolds number flows are possible with reasonable computational efforts. In contrast to a RANS calculation, where all turbulent scales in the flow are modelled and only a time averaged flow field is obtained, DNS or LES directly provides information about turbulent quantities and sources of noise. To be able to improve existing CFD routines for more reliable results one needs to increase the understanding of noise source

\footnotetext{
${ }^{1}$ Jet Exhaust Aerodynamics and Noise, contract number G4RD-CT2000-000313
}

mechanisms. Therefore, it is of great importance to perform more detailed calculations such as DNS or LES to get a more realistic picture of the flow physics. This is the main object of the present work.

LES of a Mach 0.75 nozzle/jet configuration has been performed. The Reynolds number based on the nozzle exit diameter and the jet velocity at the nozzle exit plane, $R e_{D}$, was 50000 . The Reynolds number in the measurements ${ }^{1-3}$ is approximately one million. Such a high Reynolds number probably means that the scales needed to be resolved are too small. Thus the Reynolds number in our LES was decreased with the assumption that the flow is only weakly Reynolds number dependent. An unheated jet was simulated, i.e. the static temperature in the nozzle exit plane, $T_{j}$, was equal to the static temperature of the ambient air, $T_{\infty}$. The time averaged flow field has been compared with experimental data provided by Laboratoire d'Etude Aeródynamiques, Poitiers. ${ }^{1}$ The nozzle used in the simulation corresponds to the last contraction of the nozzle configuration in the experimental setup.

\begin{tabular}{||l|l||}
\hline$U_{j} / c_{\infty}$ & 0.75 \\
\hline$T_{j} / T_{\infty}$ & 1.0 \\
\hline$P_{\infty}$ & $101300[\mathrm{~Pa}]$ \\
\hline$\rho_{\infty}$ & $1.2256\left[\mathrm{~kg} / \mathrm{m}^{3}\right]$ \\
\hline$c_{\infty}$ & 340.174 \\
\hline$U_{\infty}$ & $0.0[\mathrm{~m} / \mathrm{s}]$ \\
\hline$T_{\infty}$ & $288[\mathrm{~K}]$ \\
\hline$T_{0_{j}}$ & $320.4[\mathrm{~K}]$ \\
\hline
\end{tabular}

Table 1 Flow properties

Two-point space-time correlations have been obtained for a few locations in the shear layer. From these estimates of integral length and time scales as well as eddy convection velocities have been evaluated. Two point measurements in the shear layer at $x=L_{c}$ and $x=0.75 L_{c}$ by Jordan et al. ${ }^{2}$ have been used for comparison.

Evaluation of far-field sound pressure levels has been performed using Kirchhoff surface integration. Predicted sound pressure levels have been compared with experimental results. ${ }^{3}$

\section{Governing equations}

The equations solved are the spatially Favre filtered continuity, momentum and energy equations,

$$
\begin{gathered}
\frac{\partial \bar{\rho}}{\partial t}+\frac{\partial\left(\bar{\rho} \tilde{u}_{i}\right)}{\partial x_{i}}=0 \\
\frac{\partial\left(\bar{\rho} \tilde{u}_{i}\right)}{\partial t}+\frac{\partial\left(\bar{\rho} \tilde{u}_{i} \tilde{u}_{j}\right)}{\partial x_{j}}=-\frac{\partial \bar{p}}{\partial x_{i}}+\frac{\partial \bar{\sigma}_{i j}}{\partial x_{j}}+\frac{\partial \tau_{i j}}{\partial x_{j}} \\
\frac{\partial\left(\bar{\rho} \tilde{e}_{0}\right)}{\partial t}+\frac{\partial\left(\bar{\rho} \tilde{e}_{0} \tilde{u}_{j}\right)}{\partial x_{j}}=-\frac{\partial \bar{p} \tilde{u}_{j}}{\partial x_{j}}+ \\
+C_{p} \frac{\partial}{\partial x_{j}}\left(\left(\frac{\mu}{P r}+\frac{\mu_{t}}{P r_{t}}\right) \frac{\partial \tilde{T}}{\partial x_{j}}\right)+\frac{\partial}{\partial x_{j}} \tilde{u}_{i}\left(\bar{\sigma}_{i j}+\tau_{i j}\right)
\end{gathered}
$$


where $\bar{\sigma}_{i j}$ and $\tau_{i j}$ are the Favre filtered viscous stress tensor and sub-grid scale viscous stress tensor respectively. These are here given by

$$
\begin{gathered}
\bar{\sigma}_{i j}=\mu\left(2 \tilde{S}_{i j}-\frac{2}{3} \tilde{S}_{m m} \delta_{i j}\right) \\
\tau_{i j}=\mu_{t}\left(2 \tilde{S}_{i j}-\frac{2}{3} \tilde{S}_{m m} \delta_{i j}\right)-\frac{2}{3} \bar{\rho} k^{S G S} \delta_{i j}
\end{gathered}
$$

where $k^{S G S}$ is the sub-grid scale kinetic energy

$$
k^{S G S}=C_{I} \Delta^{2} \tilde{S}_{m n} \tilde{S}_{m n}
$$

$\mu_{t}$ the sub-grid scale kinematic viscosity

$$
\mu_{t}=C_{R} \bar{\rho} \Delta^{2}\left(\tilde{S}_{m n} \tilde{S}_{m n}\right)^{1 / 2}
$$

and $\tilde{S}_{i j}$ is the Favre filtered strain rate tensor defined by,

$$
\tilde{S}_{i j}=\frac{1}{2}\left(\frac{\partial \tilde{u}_{i}}{\partial x_{j}}+\frac{\partial \tilde{u}_{j}}{\partial x_{i}}\right)
$$

The filter-width used in equations 6 and 7 is the local grid cell width, i.e. $\Delta=\left(\Delta_{1} \Delta_{2} \Delta_{3}\right)^{1 / 3}$.

The sub-grid scale viscous stress tensor defined by equation 5 is a simplified version of the one used by Erlebacher et al. ${ }^{13}$ since the Leonard term has been neglected. The Smagorinsky model constants $C_{R}$ and $C_{I}$ where proposed by Erlebacher et al. ${ }^{13}$ to be

$$
\left\{\begin{array}{l}
C_{R}=0.12 \\
C_{I}=0.66
\end{array}\right.
$$

The system of governing equations, equations 13 , is closed by making assumptions concerning the thermodynamics of the gas considered. It is assumed that the gas is a thermally perfect gas, i.e. it obeys the gas law.

$$
\bar{p}=\bar{\rho} R \tilde{T}
$$

Furthermore, the gas is assumed to be calorically perfect, which implies that internal energy and enthalpy are linear functions of temperature.

\section{Kirchhoff Surface Integral Formulation}

Kirchhoff integration is a method to predict the value of a property, $\Phi$, governed by the wave equation in a point outside a surface enclosing all generating structures, Lyrintzis. ${ }^{14}$

$\Phi(\mathbf{x}, t)=\frac{1}{4 \pi} \int_{S}\left[\frac{\Phi}{r^{2}} \frac{\partial r}{\partial n}-\frac{1}{r} \frac{\partial \Phi}{\partial n}+\frac{1}{c_{\infty} r} \frac{\partial r}{\partial n} \frac{\partial \Phi}{\partial t}\right]_{\tau_{r}} d S(\mathbf{y})$

$\tau_{r}$ denotes that the expression within brackets is to be evaluated at retarded time, i.e. emission time. $\tau_{r}$ is related to the observer evaluation time, $t$, as

$$
\tau_{r}=t-\frac{r}{c_{\infty}}
$$

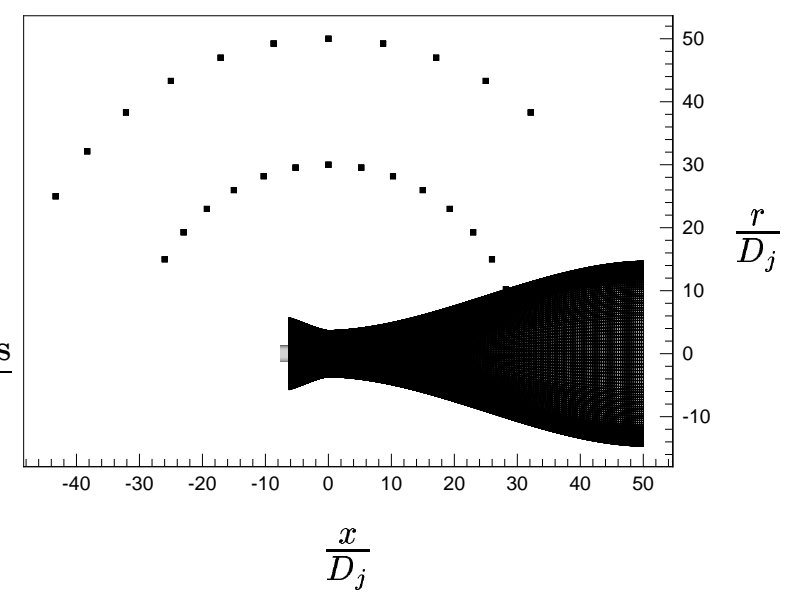

Fig. 1 Kirchhoff surface and far-field observer locations. The lower set of observer points are located on a $30 D_{j}$ radius circle, with origin at the nozzle exit, and the upper points on a $50 D_{j}$ radius circle.

where $r$ is the distance between a point on the surface, $\mathbf{y}$, and an observer location, $\mathbf{x} . c_{\infty}$ is the speed of sound in the far-field region. The variable, $\Phi$, to be evaluated, is in this case the surface pressure, $S$ is the surface enclosing all sound generating structures and $n$ denotes the direction normal to the surface. The surface, $S$, must be placed in a region where the flow is completely governed by a homogeneous linear wave equation with constant coefficients, Freund et al. ${ }^{10}$ More details about the Kirchhoff surface integration method, can be found in e.g. Freund et al. ${ }^{10}$ and Lyrintzis. ${ }^{14}$

Since the hydrodynamic source region decays slowly downstream it is not possible to use a surface enclosing the entire source region without entering this non-linear region. Therefore, it is common practice to use Kirchhoff surfaces not closed in the upstream and downstream ends. It has been shown by Freund et al. ${ }^{10}$ that the errors introduced by using such surface are small if the main part of sources of sound is within the axial extent of the surface and if lines connecting observer points with points in the hydrodynamic region, representing the main sources of sound, intersects with the surface.

In this work a Kirchhoff surface closed in the upstream end and open in the downstream end have been used, see figure1.

\section{Numerical method}

\section{Numerical scheme}

The governing equations are solved using a cell centered finite volume method solver. A low dissipative third order upwind scheme is used for discretization of the convective fluxes and a second order central difference approach for the viscous fluxes. Time marching is performed using a second order three-stage Runge-Kutta technique. For more details see Eriksson. ${ }^{15}$ 


\section{Computational domain}

The computational domain consists of a boundary fitted block structured mesh with 50 blocks and a total of about 3000000 cells, figures $2-4$. The grid cells are concentrated to the shear layer area. To establish mesh homogeneity a combination of polar and cartesian blocks, figure 4 , has been used. The grid cells are stretched in the downstream direction and radially outwards the boundaries using cubic Hermite grid point distribution. The last contraction of the JEAN project nozzle is included in the calculation domain. The axial extent of the physical part of the domain is 2.5 meters, which is equal to 50 nozzle diameters $\left(D_{j}=50[\mathrm{~mm}]\right)$. The radial extent is 10 nozzle diameters at the nozzle exit plane and 20 diameters at the domain outlet, see figure 5 .

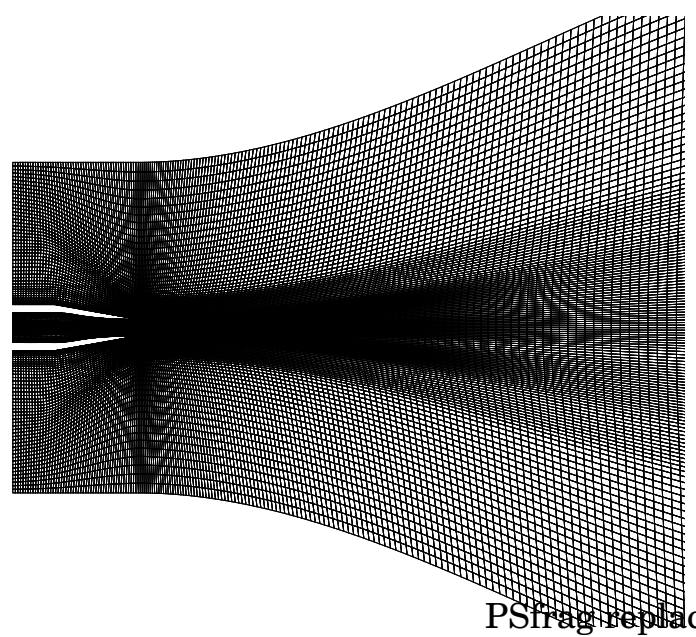

Fig. 2 A slice through the calculation domain made at $z=0$, i.e. a $x y$-plane, is depicted. The picture shows the domain inlet including the nozzle and the outer boundaries in the radial direction. About half of the domain axial extent is shown in the figure.

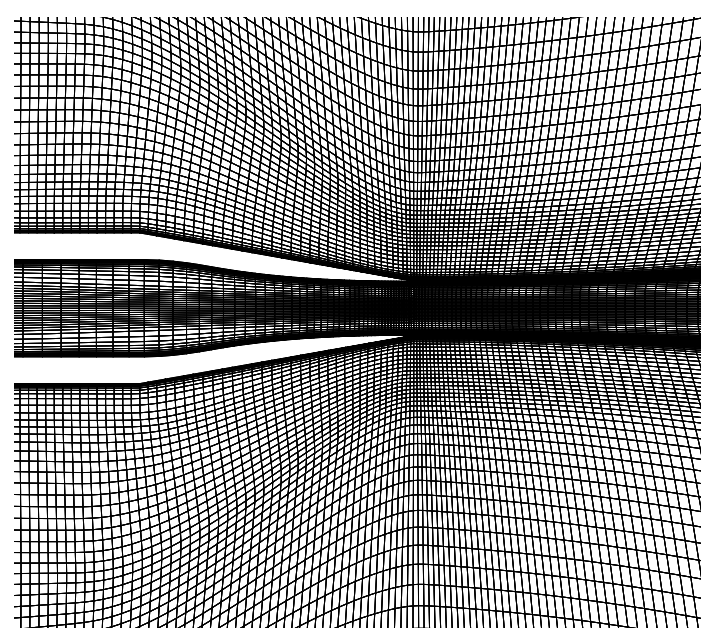

Fig. 3 A slice of the calculation domain in the nozzle region is shown. Cells are concentrated to the shear layer area.

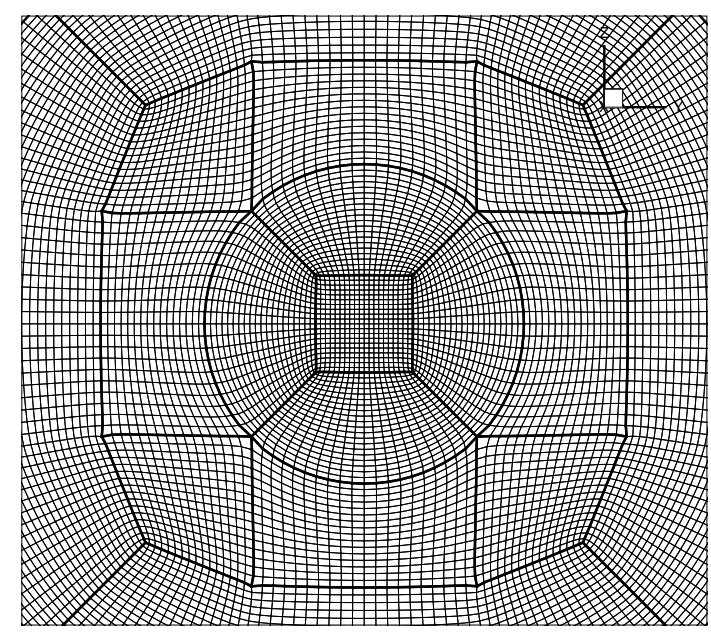

Fig. 4 A slice through the calculation domain at constant $x$, i.e. a $y z$-plane, is depicted. Combining cartesian and polar grid blocks enhance the radial direction grid homogeneity throughout the domain.

\section{Boundary conditions}

At the inlet of the nozzle, the nozzle plenum, total pressure and total enthalpy are specified. All free boundaries, i.e. upstream and entrainment boundaries are defined using absorbing boundary conditions based on characteristics variables. At the domain outlet static pressure is specified. In figure 5 the main boundary condition locations are depicted.

entrainment boundaries outlet

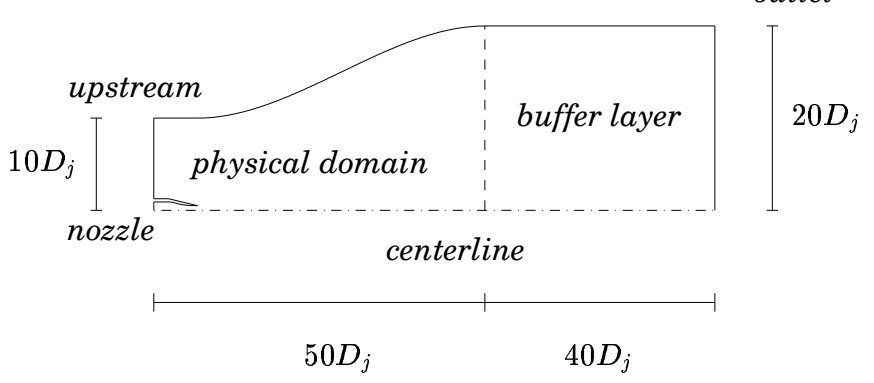

Fig. 5 Computational setup. $D_{j}=50[\mathrm{~mm}]$.

Defining proper boundary conditions is an issue of great importance, especially in aeroacoustic applications since acoustic pressure fluctuations are small and spurious waves generated at the boundaries might ruin the results. Definition of boundary conditions for free shear flows are particularly difficult since there are, by definition, no bounding surfaces. The difficulties of boundary definitions for free shear flows have been discussed in several publications, e.g. Bogey et al., ${ }^{16}$ Colonius et al. ${ }^{17}$ and Mankbadi et al. ${ }^{7}$

At a finite distance downstream the jet, bound- 
ary conditions that mimic the jet behavior at infinity are to be specified. Energetic vorticity and entropy waves traveling out of the domain reaching the outlet boundary will, if not damped out, generate strong acoustic waves traveling back into the domain. These acoustic reflections can be diminished by, in some way, decreasing the amplitude of fluctuations of vorticity and entropy waves approaching the boundary. Inward traveling acoustic waves may still be generated but will be weaker than for a boundary where no special treatment of the boundary region is utilized. Moreover, these weaker acoustic waves are additionally damped when traveling through the outlet region on their way back into the calculation domain and hopefully the waves are so weak when reaching the jet region that they do not affect the results. Therefore, an extra outlet zone has been added to the calculation domain, figure 5 , where a damping term defined by

$$
\varepsilon(Q-\langle Q\rangle)
$$

has been added to the equation system. $\varepsilon$ is defined by a constant $\varepsilon_{\max }$ and the axial location, $x$, in the damping zone as

$$
\varepsilon=\varepsilon_{\max }\left(\frac{x-x_{\min }}{x_{\max }-x_{\min }}\right)^{2}
$$

In equation $13, Q$ represents the flow variables and the time average of $Q$ is calculated as

$$
\langle Q\rangle=\frac{\sum_{i=1}^{n} Q_{i} t_{i}}{\sum_{i=1}^{n} t_{i}}
$$

where subscript $i$ denotes time step. The weighted time average defined by equation 15 gives the recent flow property values higher weight than older values, but at the same time gives a good estimate of the time averaged flow field. The fluctuations of the flow field are damped in the boundary zone by forcing the flow properties towards the time averaged flow field. Furthermore, the cells in this part of the calculation domain are more stretched than cells in the physical part of the calculation domain, which increases the numerical dissipation and thereby flow field fluctuations are further damped. The boundary zone consists of roughly 500000 cells, which is about $14 \%$ of the total calculation domain, and has an axial extent of two meters, see figure 5 .

The entrainment boundaries proved to be rather troublesome as well. The problem is to get enough fluid entrainment into the domain. The effect of not getting the entrained mass flow correct is that a deficit of mass is compensated with inflow of fluid from the domain outlet. This back flow results in a recirculation zone surrounding the jet and prevents it from spreading. Approximate values of the velocities specified at the entrainment boundaries were obtained from RANS calculations ${ }^{18}$ with $U_{\infty}=0$.
These RANS calculations have been performed using significantly larger calculation domain than in the LES and therefore give reliable information on the entrainment effect at the boundary of the LES domain. Furthermore, these RANS calculations have been have been extensively validated against experimental data. ${ }^{1}$ The fact that the boundary values at the entrainment boundaries have been obtained from RANS results does, to a certain degree, determine the time-averaged flow field. Since this work is not an attempt to prove that Large Eddy Simulation predicts the flow of a jet properly but rather to obtain a database of flow properties for jets and to get input for calculations of the acoustic field this is no severe disadvantage.

\section{Results}

Profiles of statistic quantities shown in figures below are obtained from flow field data that have been averaged in both time and the azimuthal direction to establish improved statistical convergence. The profiles are made non-dimensional using the jet velocity at the nozzle exit, $U_{j}$, and the nozzle outlet diameter, $D_{j}$.

The calculations were started up using an initial solution interpolated from RANS. The simulation was running for about 100000 time steps before sampling were initiated. This corresponds to 0.025 seconds of simulated time using a time step of $0.25[\mu s]$ or roughly 3.5 acoustic through-flows, i.e. the time required for an acoustic wave to travel through the calculation domain, not including the outlet buffer layer, based on the speed of sound at ambient conditions. Sampling of statistic data was then continued for another 20 acoustic throughflows. Each acoustic through-flow corresponds to roughly 32 hours of computer wall-time using 14 AMD $1700^{+}$processors of our Linux-cluster. The time step is chosen such that the $\mathrm{CFL}^{2}$ number is nowhere in the calculation domain larger than 0.5.

\section{Time averaged profiles}

In figure 6 the development of radial profiles of axial velocity downstream the nozzle exit is depicted.

Comparison of profiles obtained from LES and experiments are presented in figures 7-13. The radial profiles have been staggered corresponding to axial position, i.e. 1.0, 2.5 and 5.0 jet diameters respectively, to show downstream development. Furthermore, it should be noted that, in order to obtain similar scales for all the radial profile figures, root-mean-square values and uv-correlations have been multiplied by a factor 4 and 70 respectively. Although the LES results are in good agreement with experiments, there are some deviations. The main discrepancy is that the LES fail to predict the length of the jet potential core, figure 7 , and

\footnotetext{
${ }^{2}$ Courant-Friedrichs-Lewy
} 


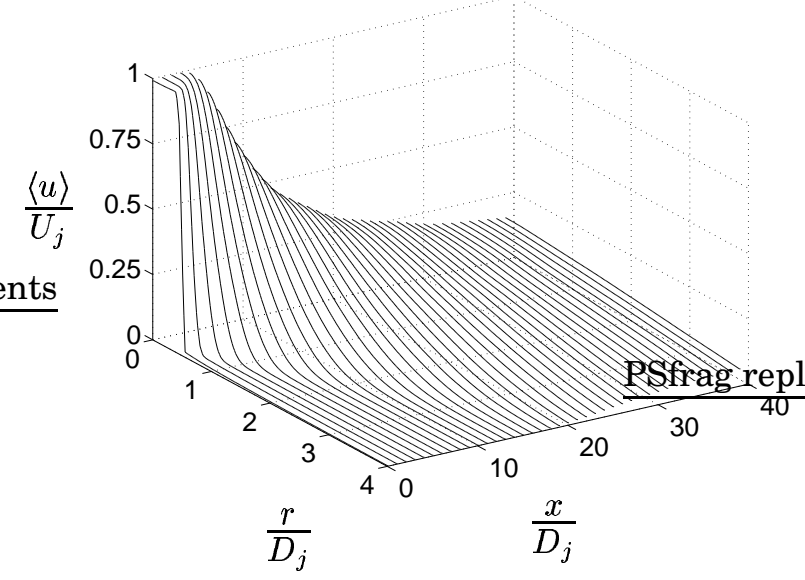

Fig. 6 Downstream development of radial profiles of axial velocity.

thereby the location of maximum turbulent kinetic energy, figure 9. It has been mentioned by DeBonis et al. ${ }^{19}$ that this is generally not the case in earlier studies of jets, rather the length of the potential core is usually over predicted. Although the location of the potential core closure is not accurately predicted the decay of centerline axial velocity is. Moreover, it is seen from figure 8 that the jet spreading is under-predicted. This is probably an effect of the entrainment boundary conditions used. Moreover, it has been mentioned by Morris et al. ${ }^{20}$ that a change of the SGS model constants affect the potential core length and the initial growth of the jet. These sub-grid scale effects can, since the grid used for the simulation is rather coarse, probably be diminished by grid refinement or by use of a more sophisticated sub-grid scale model. Large deviations from experimental data in root-meansquare values of radial velocity near the centerline, figure 11-12, might be due to low frequency flutter of the potential core found in the experiments. ${ }^{1}$ Centerline profiles of root-mean-square profiles of axial and radial velocity are shown in figure 9 and figure 11 respectively.

\section{Self-preservation}

Radial profiles of axial velocity and second order moments have been normalization by centerline velocity and plotted versus non-dimensional radial coordinate to investigate whether self-preservation has been established or not. These profiles are obtained using statistical flow field data averaged in time and the azimuthal direction. The constant $x_{0}$ used to collapse the profiles is an imaginary origin obtained from the least square fit of data points in figure 14, i.e. the axial position for which $U_{j} /\left\langle u_{c}\right\rangle_{t}=0$. In this case $x_{0}=0.11 D_{j}$. As seen from figure 14 a non-negligible deviation from the linear relation occurs for centerline points located between $20 D_{j}$ and $35 D_{j}$ downstream the nozzle exit. This behavior is probably due to the boundary conditions used. However, since this occurs

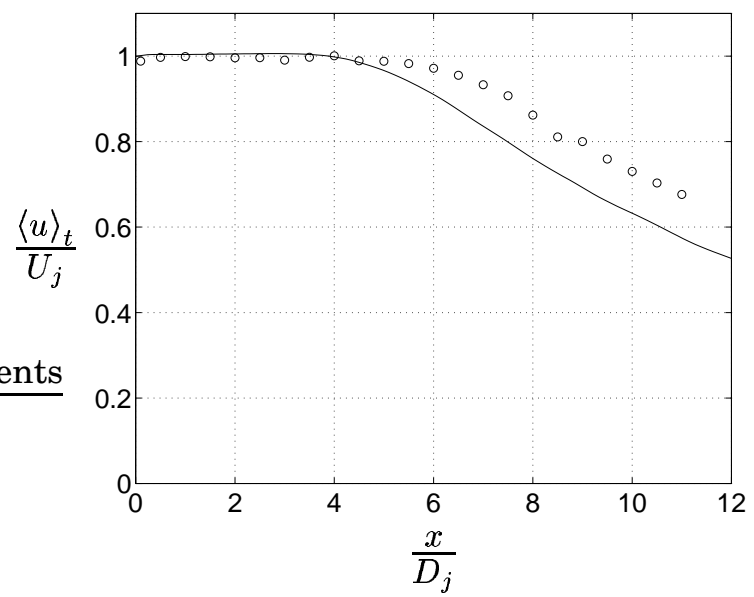

Fig. 7 Center line velocity: the solid line, ' - ', corresponds to LES results and the circles, ' $o$, to experimental data. ${ }^{1}$

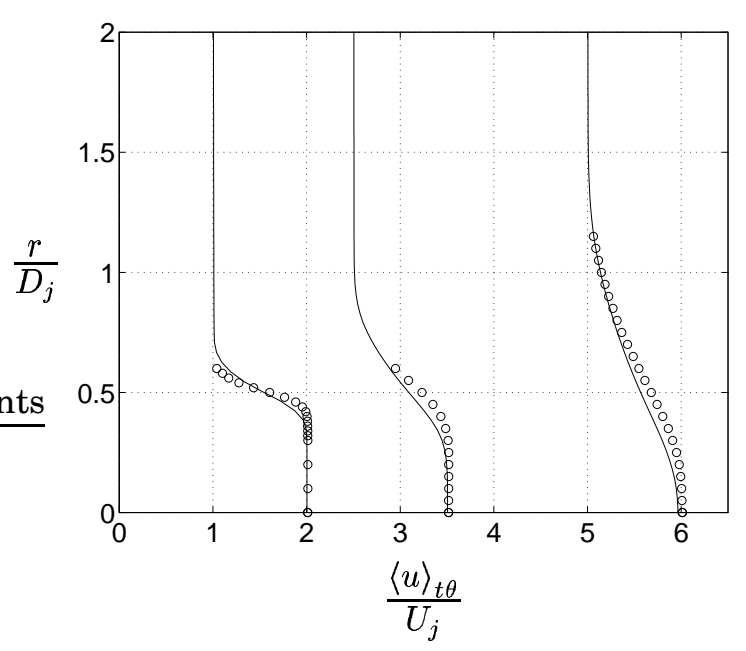

Fig. 8 Radial profiles of axial velocity at the axial positions $x / D_{j}=1.0, x / D_{j}=2.5$ and $x / D j=5.0$ : ' - ' corresponds to LES results and ' $o$ ' to experimental data. ${ }^{1}$ The profiles have been staggered corresponding to their axial position.

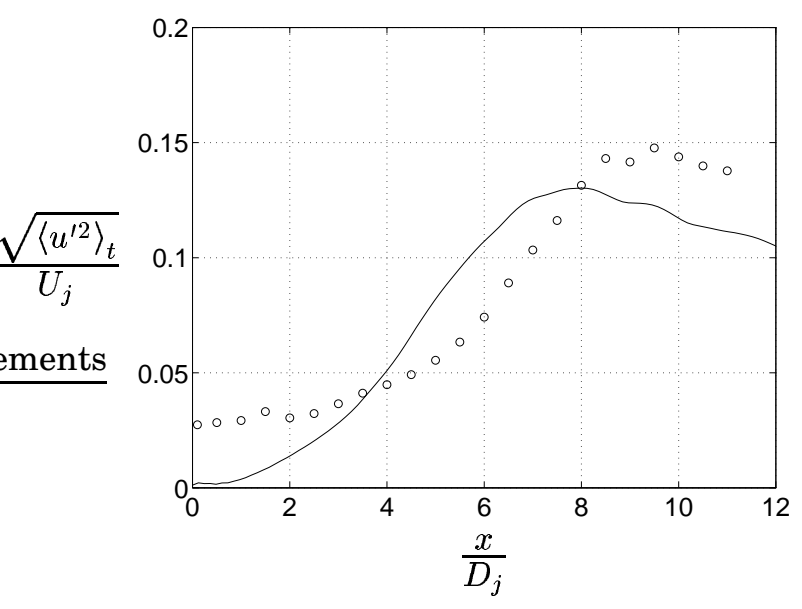

Fig. 9 Axial profile of $\sqrt{\left\langle u^{\prime 2}\right\rangle_{t}}:{ }^{\prime}{ }^{\prime}$ corresponds to LES results and ' $o$ ' to experimental data. ${ }^{1}$ 


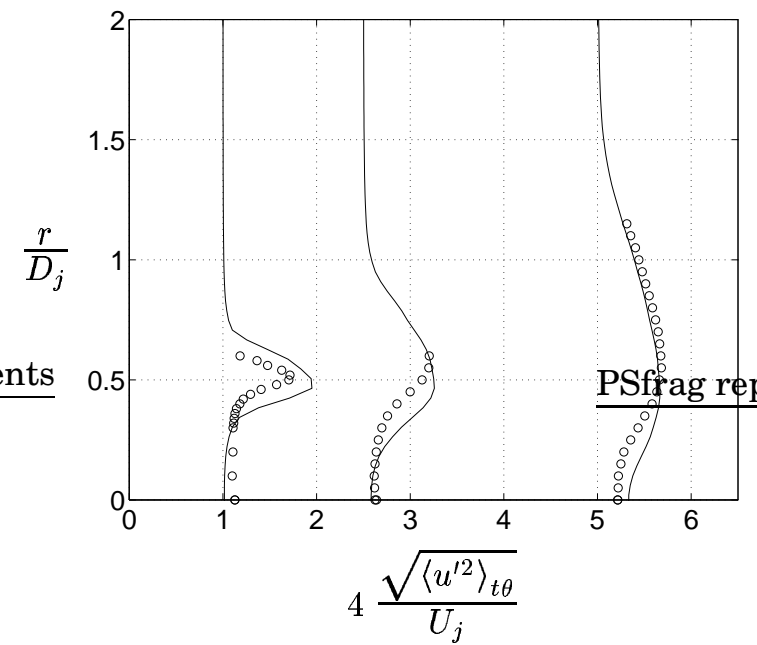

Fig. 10 Radial profiles of $\sqrt{\left\langle u^{\prime 2}\right\rangle_{t \theta}}$ at $x / D_{j}=1.0$, $x / D_{j}=2.5$ and $x / D_{j}=5.0:^{\prime}{ }^{\prime}$ corresponds to LES results and ' $o$ ' to experimental data. ${ }^{1}$ The profiles have been staggered corresponding to their axial position.

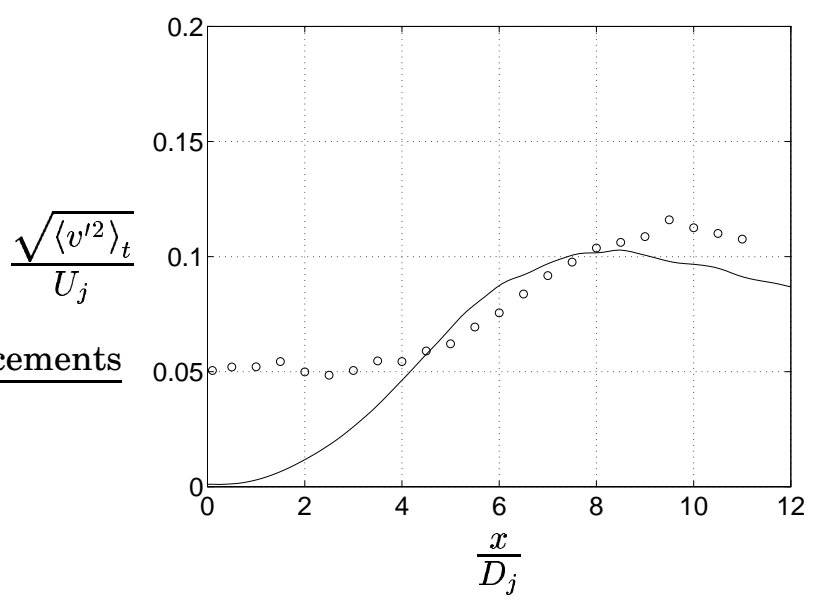

Fig. 11 Axial profile of $\sqrt{\left\langle v^{\prime 2}\right\rangle_{t}}:{ }^{\prime}{ }^{\prime}$ corresponds to LES results and ' $o$ ' to experimental data. ${ }^{1}$

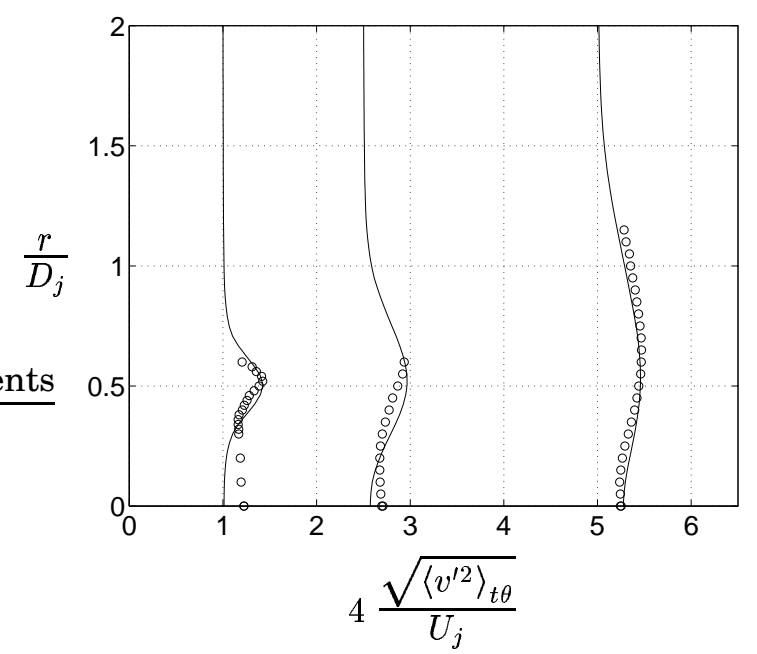

Fig. 12 Radial profiles of $\sqrt{\left\langle v^{\prime 2}\right\rangle_{t \theta}}$ at $x / D_{j}=1.0$, $x / D_{j}=2.5$ and $x / D_{j}=5.0$ : $^{\prime}-{ }^{\prime}$ LES and ' $o$ ' to experimental data. ${ }^{1}$ The profiles have been staggered corresponding to their axial position.

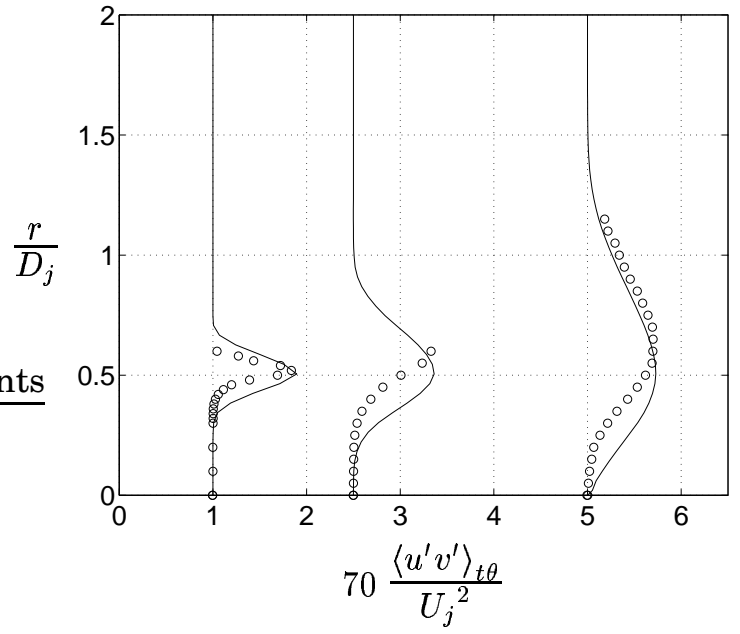

Fig. 13 Radial profiles of $\left\langle u^{\prime} v^{\prime}\right\rangle_{t \theta}$ at $x / D_{j}=1.0$, $x / D_{j}=2.5$ and $x / D_{j}=5.0$ : $^{\prime}-{ }^{\prime}$ corresponds to LES and ' $o$ ' to experimental data. ${ }^{1}$ The profiles have been staggered corresponding to their axial position.

far downstream, the effect on the flow field in the potential core region is probably negligible. In figure 15 collapsed radial profiles of axial velocity are shown. Five radial profiles equally spaced between $19 D_{j}$ and $39 D_{j}$ have been used. The jet-spreading rate can be estimated to be roughly 0.09 based on the curve fitted to the LES data. In figures 16-19 normalized profiles of second order moments are depicted. For these figures radial profiles in six axial positions from $29 D_{j}$ to $39 D_{j}$ downstream the nozzle have been used. The distance between each profile is, in this case, two jet diameters. As can be seen from the figures 15-19 self-preservation of axial velocity has been well established within 39 jet diameters downstream the nozzle whereas for self-preservation of second order moments to be obtained data would have to be extracted further downstream. For example Hussein et al. ${ }^{21}$ found that profiles of second and higher order moments displayed self-similar behavior when evaluated at axial positions more than $70 D_{j}$ downstream the imaginary origin. However, this would require a larger computational domain to be used.

\section{Two-point space-time correlations}

Two-point space-time correlations have been obtained for axial velocity in a few points in the shear layer region downstream the nozzle exit. The time series used is 0.0725 seconds long. Spatial separations, $\xi$, are, in this case, downstream in the axial direction. The two-point space-time correlation of axial velocity for a certain spatial separation, $\xi$, and separation in time, $\tau$, is given by

$$
R_{u u}(\mathbf{x}, \xi, \tau)=\frac{\left\langle u^{\prime}(\mathbf{x}, t) u^{\prime}(\mathbf{x}+\xi, t+\tau)\right\rangle_{t}}{\sqrt{\left\langle u^{\prime 2}(\mathbf{x})\right\rangle_{t}} \sqrt{\left\langle u^{\prime 2}(\mathbf{x}+\xi)\right\rangle_{t}}}
$$

where $u^{\prime}$ denotes fluctuation of axial velocity and $\mathbf{x}$ is the position in the flow field where the two-point 


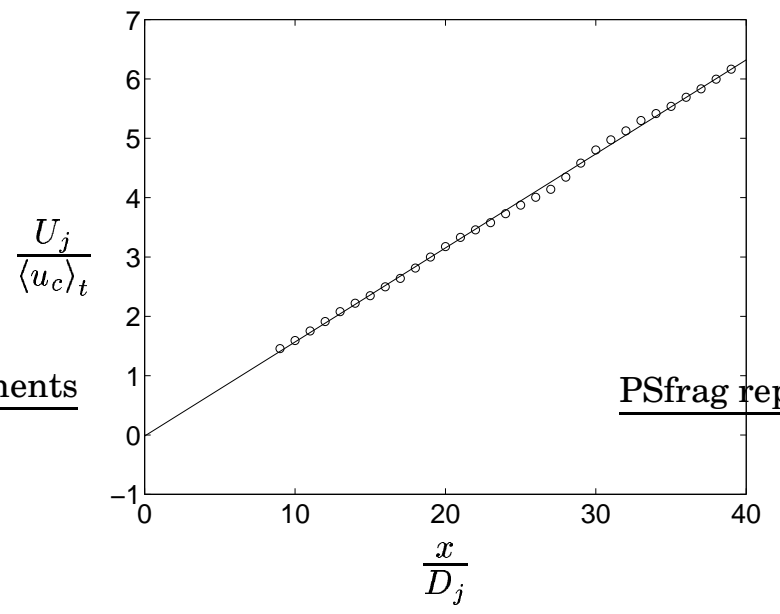

Fig. 14 Predicted center line velocity. The solid line corresponds to a curve fit to the LES data. The $x$-value for which the line intersects with the $x$-axis, $x_{0}$, is used to collapse the radial profiles in figures 15-19.

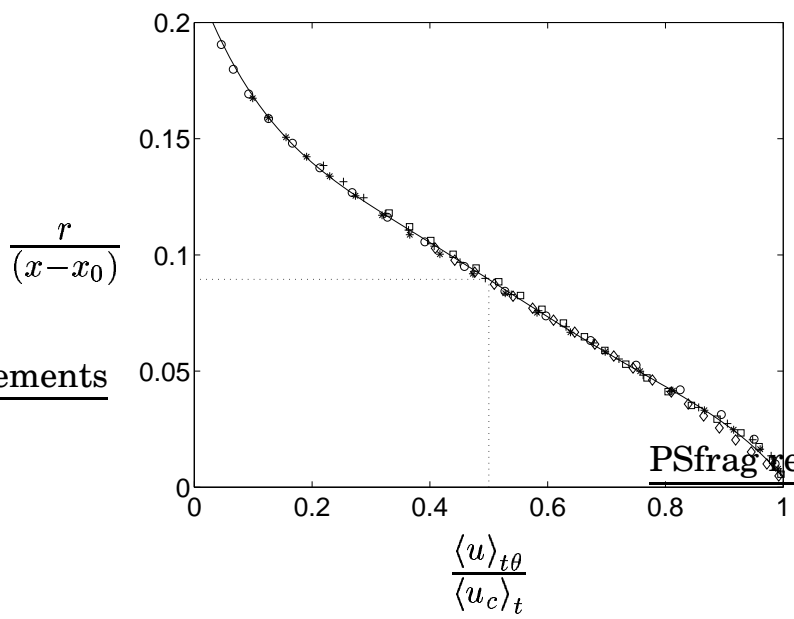

Fig. 15 Radial profiles of axial velocity normalized by centerline mean velocity. The solid line corresponds to a curve fit to the LES data. The profiles are labeled as follows: ' $\circ^{\prime}=19 D_{j},{ }^{\prime}{ }^{\prime}=24 D_{j}$, ${ }^{\prime}+{ }^{\prime}=29 D_{j},{ }^{\prime} \square^{\prime}=34 D_{j}$ and ${ }^{\prime} \diamond^{\prime}=39 D_{j}$.

correlation is evaluated.

In figures 20-22 two-point correlations in points located on the nozzle lip-line 2.5, 5.0 and 10.0 nozzle diameters downstream the nozzle exit are presented. Each figure contains the autocorrelation of axial velocity fluctuations, i.e. no spatial separation, and 30 two-point space-time correlations obtained with increased spatial separation. The spatial separation is increased by $0.1 D_{j}$ for each correlation curve. The integral of the envelope built up of the cross-correlation curves corresponds to the Lagrangian time scale in the current position in the flow. Comparing the figures 20-22, the amplitude for a given spatial separation, $\xi$, increases as moving downstream the jet, which indicates that the turbulence becomes more and more frozen in character, i.e. the turbulence length scale increases, as the jet develops. Comparison has been made with

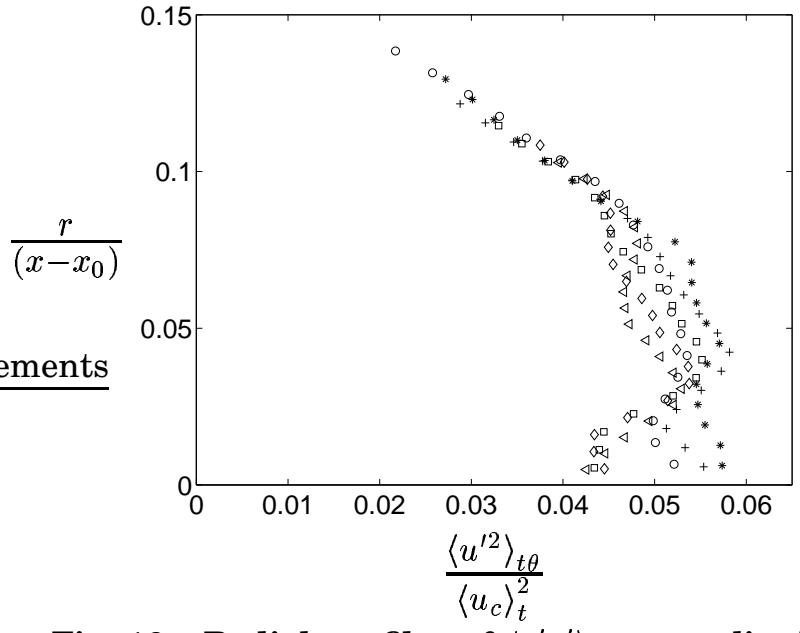

Fig. 16 Radial profiles of $\left\langle u^{\prime} u^{\prime}\right\rangle_{t \theta}$ normalized by centerline mean velocity. Radial profiles at six axial locations are represented. The profiles are labeled as follows: ${ }^{\prime} \circ{ }^{\prime}=29 D_{j},{ }^{\prime} *^{\prime}=31 D_{j}$, $'+{ }^{\prime}=33 D_{j},{ }^{\prime} \square^{\prime}=35 D_{j},{ }^{\prime} \diamond^{\prime}=37 D_{j}$ and finally $\triangleleft^{\prime}=39 D_{j}$.

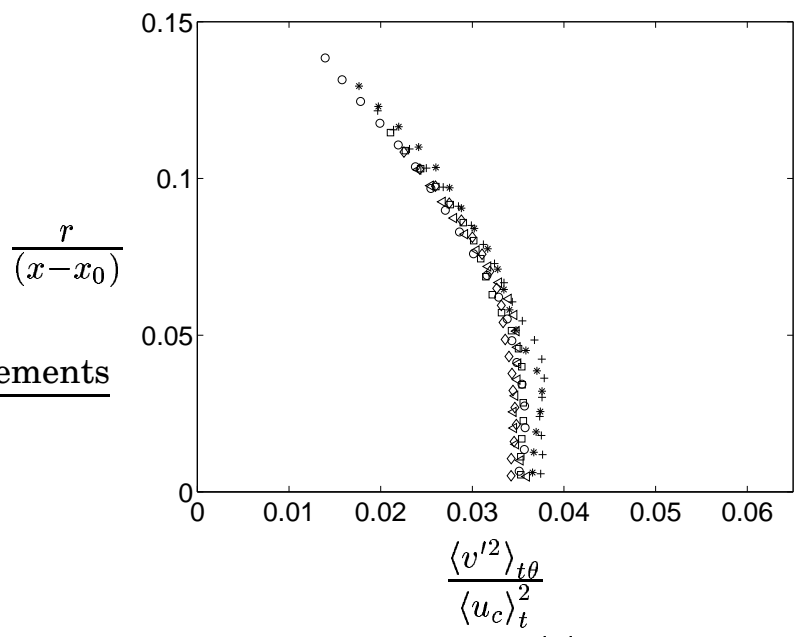

Fig. 17 Radial profiles of $\left\langle v^{\prime} v^{\prime}\right\rangle_{t \theta}$ normalized by centerline mean velocity. For legend see figure 16.

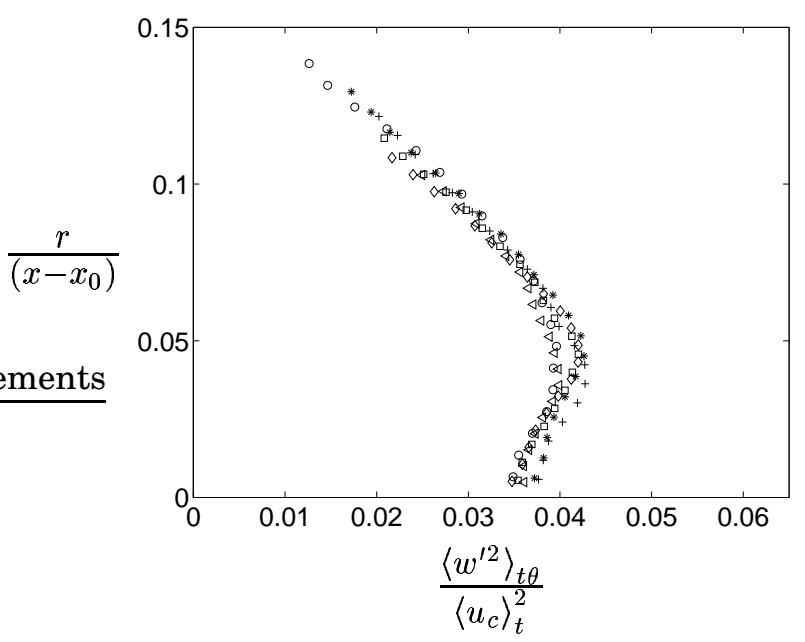

Fig. 18 Radial profiles of $\left\langle w^{\prime} w^{\prime}\right\rangle_{t \theta}$ normalized by centerline mean velocity. For legend see figure 16. 


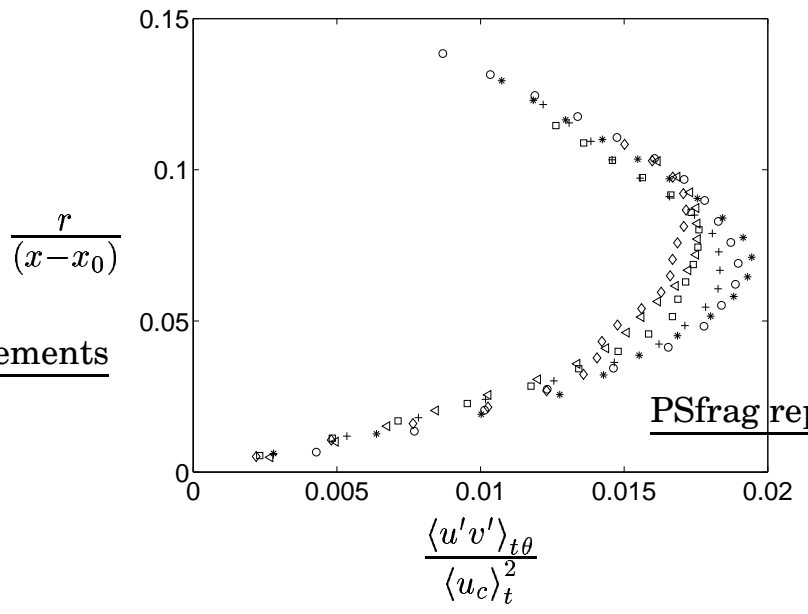

Fig. 19 Radial profiles of $\left\langle u^{\prime} v^{\prime}\right\rangle_{t \theta}$ normalized by centerline mean velocity. For legend see figure 16.

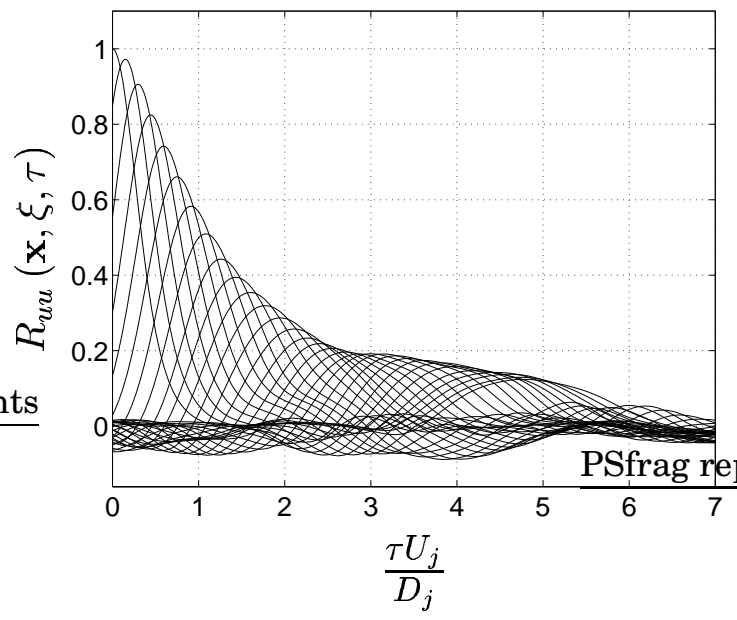

Fig. 20 Two-point space-time correlation of axial velocity at a location, $x$, in the shear layer $r=0.5 D_{j}, x=2.5 D_{j}$. Each curve corresponds to a two-point space-time correlation obtained using a certain spatial separation, $\xi$. Starting with no separation in space, i.e. the autocorrelation, the spatial separation is increased by $0.1 D_{j}$ per curve.

two-point measurements ${ }^{2}$ performed in the shear layer at $x=L_{c}$, where $L_{c}$ denotes the length of the potential core. Here the potential core length is defined as the axial location where the centerline mean velocity equals $0.95 U_{j}$. For the LES this gives $L_{c}=5.4545 D_{j}$. In figure 23 correlation peak locations of the cross-correlations obtained from LES data are compared with those from the two-point measurements. In figure 24 the spatial correlations of axial velocity in three locations are shown.

\section{Turbulence length and time scale}

Length and time scales of turbulence quantities have been obtained using the two-point space-time correlations presented in previous section. Estimates of convection velocity of turbulence structures have been obtained for a number of axial locations in the shear layer. The separation in time between the peak of the autocorrelation curve and the peak of the first cross-correlation curve has been

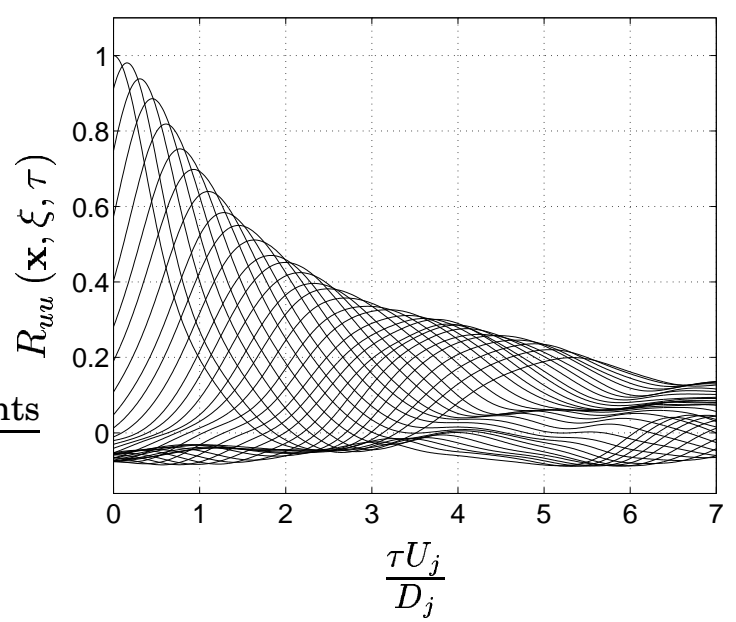

Fig. 21 Two-point space-time correlation of axial velocity at a location, $x$, in the shear layer $r=0.5 D_{j}, x=5.0 D_{j}$. See also legend in figure 20 .

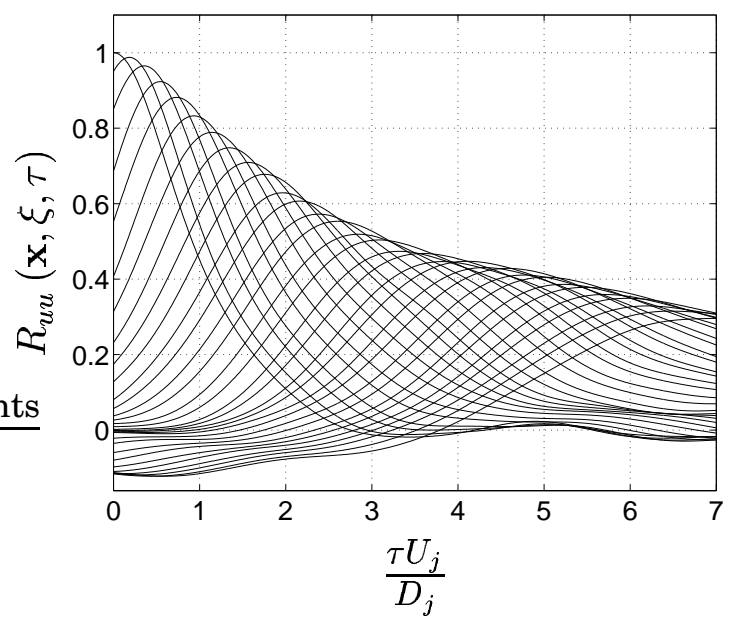

Fig. 22 Two-point space-time correlation of axial velocity at a location, $x$, in the shear layer $r=0.5 D_{j}, x=10.0 D_{j}$. See also legend in figure 20.

used. This time delay corresponds to a spatial separation of $0.1 D_{j}$ as mentioned previously, and thus gives an estimate of the convection velocity. A more correct way would be not to use the time separation for the peak but rather the time for which the crosscorrelation envelope is a tangent to the correlation curve. ${ }^{22}$ However, the result would not differ much, and predicted convection velocities for a few axial locations are presented in table 2 . In the middle column the convection velocities have been normalized by the jet velocity, $U_{j}$, and in the right column by the local mean velocity. As shown in table 2 the eddy convection velocity is everywhere higher than the local mean velocity. This might be a nonintuitive result but is a phenomenon observed in experiments ${ }^{22}$ and is due to skewness of the velocity distribution. In shear layer locations close to the high velocity potential core the convection velocity is likely to be larger than the local mean velocity and in the outer parts of the shear layer lower. ${ }^{22}$

Local integral length and time-scales are estimated by integration of corresponding autocorrela- 


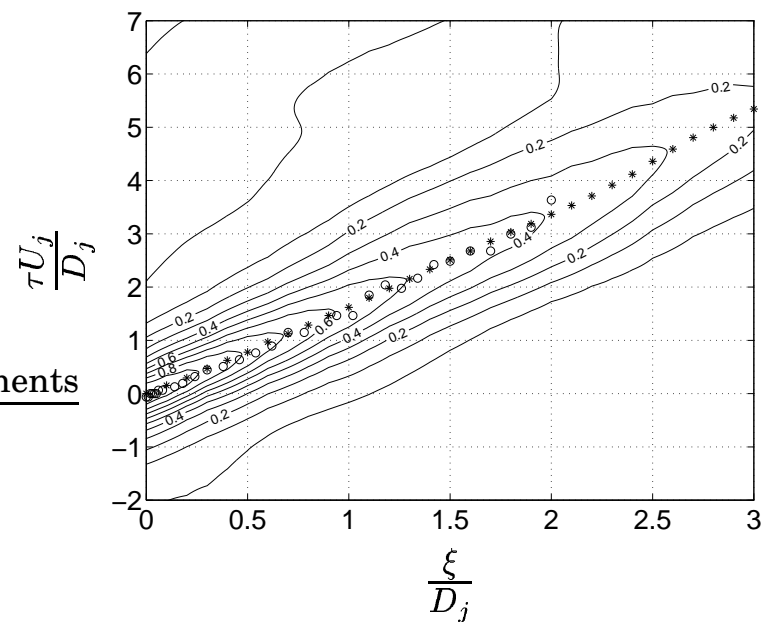

Fig. 23 Contours of $R_{u u}(\mathbf{x}, \xi, \tau)$ obtained in the shear layer, $r=0.5 D_{j}$, at the end of the potential core, $x=L_{c}=5.5 D_{j}$. The contours corresponds to LES data,' * ' corresponds to the correlation peaks of these cross-correlations and ${ }^{\prime} \circ{ }^{\prime}$ to the peaks of the cross-correlations of the measured velocity data. $^{2}$

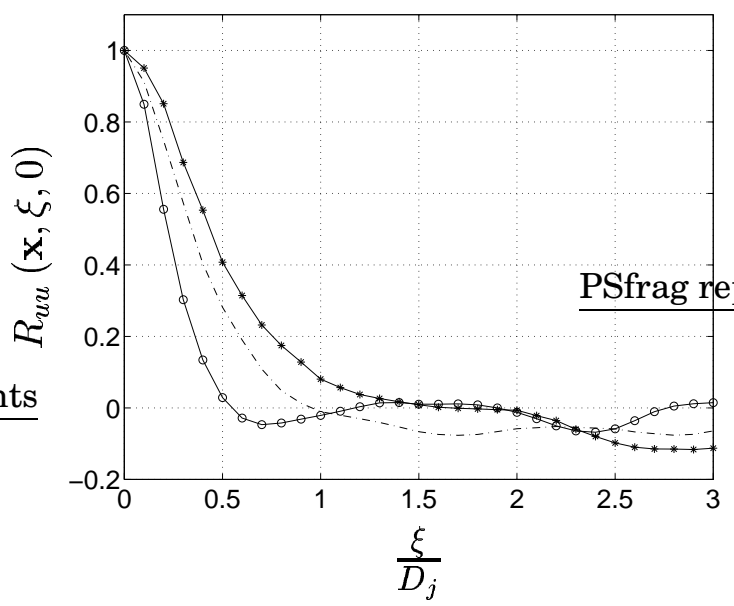

Fig. 24 Two-point space correlation of axial velocity at three different locations, $x$, in the shear layer: ${ }^{\prime}-o$ ' corresponds to a location $x=2.5 D_{j}$ downstream the nozzle exit, ' $-{ }^{\prime}$ to $x=5.0 D_{j}$ and ${ }^{\prime}-*^{\prime}$ to $x=10 D_{j}$. All points are located at $r=0.5 D_{j}$.

tion and spatial correlation, respectively. The first crossing of the coordinate axis is used as the upper limit for the integration. Axial development of integral length and time scales are presented in figures 25 and 26 respectively.

\section{Far-field sound pressure levels}

Comparisons of predicted far-field sound pressure levels (SPL) and experimental data $^{3}$ are shown in figures 27 and 28 . The time series of far-field pressure fluctuations used for the evaluation contains about 15000 samples and is roughly $0.038[s]$ long. The observer points in figure 27 are located on a $30 D_{j}$ radius arc and in figure 28 on a $50 D_{j}$ radius arc. The observer locations in relation to the Kirchhoff surface used and the nozzle are shown in figure 1 . The sound pressure level is

\begin{tabular}{||c|c|c||}
\hline$x / D_{j}$ & $u^{c} / U_{j}$ & $u^{c} /\langle u\rangle_{t}$ \\
\hline 2.50 & 0.65 & 1.09 \\
\hline 3.00 & 0.71 & 1.19 \\
\hline 3.50 & 0.71 & 1.21 \\
\hline 4.00 & 0.71 & 1.23 \\
\hline 4.50 & 0.71 & 1.24 \\
\hline 5.00 & 0.65 & 1.15 \\
\hline 5.50 & 0.65 & 1.16 \\
\hline 6.00 & 0.60 & 1.09 \\
\hline 6.50 & 0.65 & 1.20 \\
\hline 7.00 & 0.71 & 1.31 \\
\hline 7.50 & 0.65 & 1.22 \\
\hline 8.00 & 0.65 & 1.24 \\
\hline 8.50 & 0.60 & 1.16 \\
\hline 9.00 & 0.65 & 1.28 \\
\hline 9.50 & 0.60 & 1.22 \\
\hline 10.00 & 0.56 & 1.16 \\
\hline
\end{tabular}

Table 2 Convection velocity of turbulence structures in the shear layer, $r=0.5 D_{j}$.

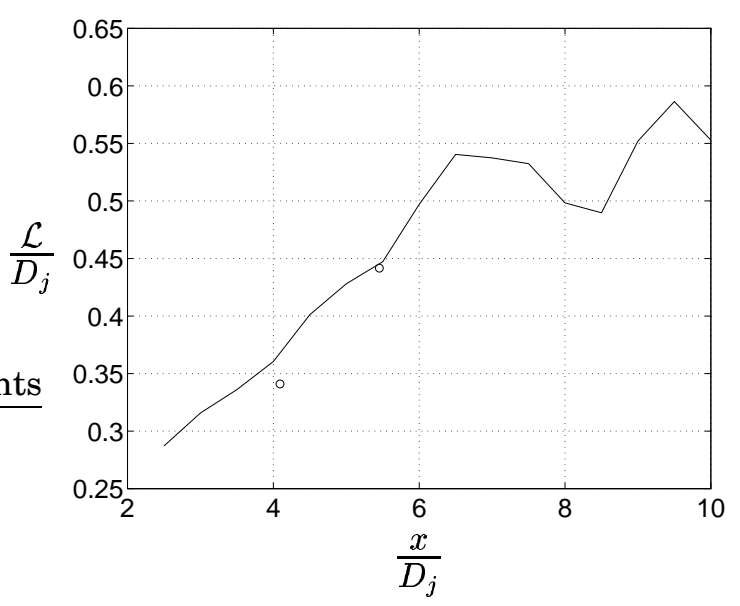

Fig. 25 Axial development of integral length scale in the shear layer, i.e. $r=0.5 D_{j} \cdot{ }^{\prime} \circ$ ' denotes experiments. $^{2}$

here defined as

$$
S P L=20 \log _{10}\left(\frac{\sqrt{\left\langle\left(p^{\prime}\right)^{2}\right\rangle_{t}}}{p_{\text {ref }}}\right)
$$

where

$$
p_{\text {ref }}=\sqrt{\rho_{\infty} c_{\infty} 10^{-12}}=2 * 10^{-5}[P a]
$$

Sound pressure levels obtained using all spectral information available are often referred to as overall sound pressure levels (OASPL). Time history of pressure in the observer locations has been obtained numerically using instantaneous flow data from the LES in combination with Kirchhoff surface integration. As can be seen in the figures, predicted levels agree very well with experimental data. The predicted levels are not as good for angles below $40^{\circ}$ and higher than $120^{\circ}$ as for those in between. This is especially noticeable for the lower arc. In this case the fact that the observer locations 


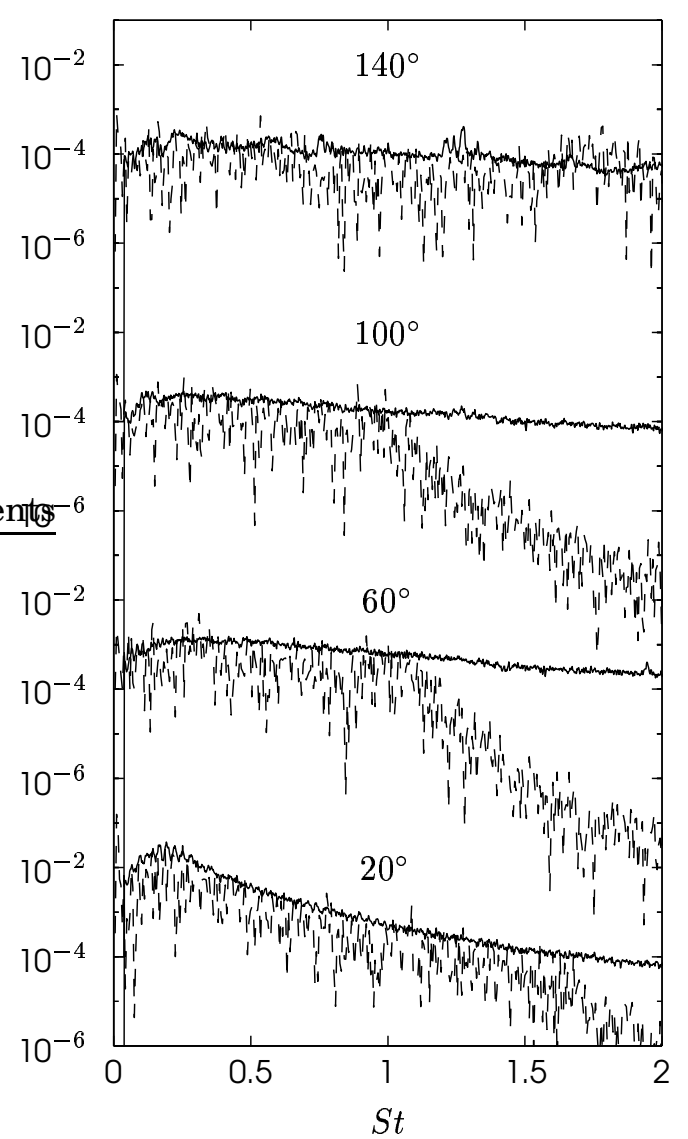

(a)

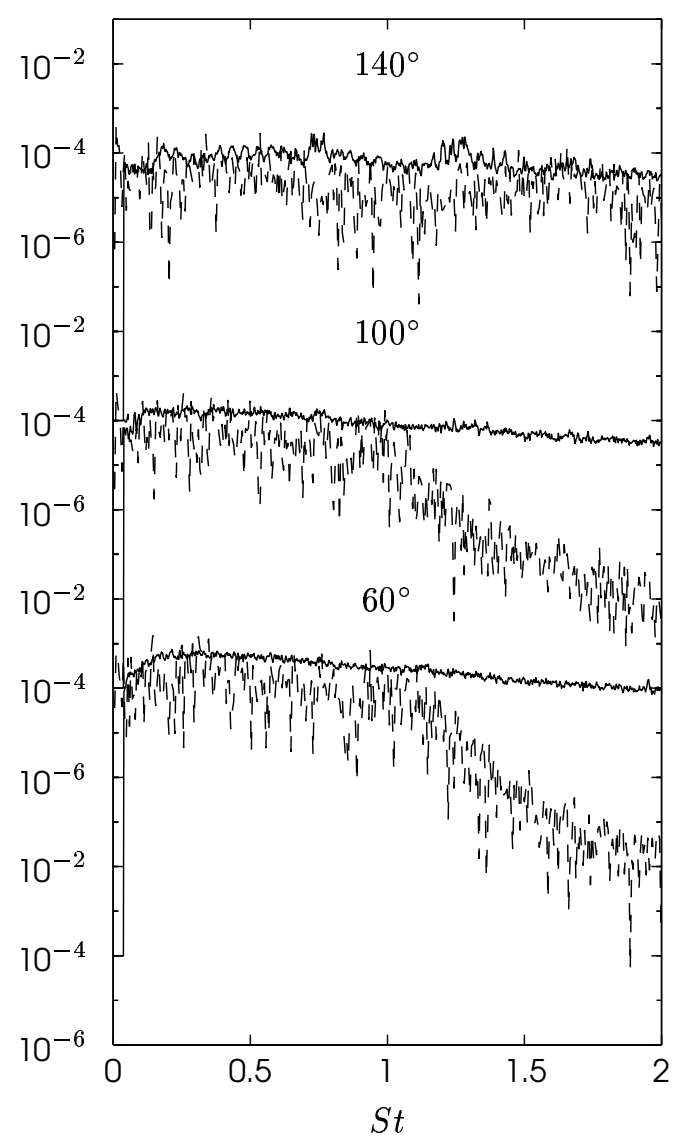

(b)

Fig. 29 Power spectrum of far-field pressure fluctuations versus Strouhal number, $S t=f D_{j} / U_{j}$. The dashed line, ' - - ' corresponds to data obtained using Kirchhoff integration and the solid line corresponds to experimental data by Jordan et al. ${ }^{3}$ Observer locations on a $30 D_{j}$ radius and $50 D_{j}$ radius circle are shown in figure (a) and figure (b), respectively. In figure (a), four observer locations are represented. Starting in a location 20 degrees from the $x$-axis the angle is increased by an increment of 40 degrees per spectrum. In figure (b) the first spectra from below is obtained for an observer location 60 degrees from the $x$-axis, i.e. it should be compared with the second spectra from below in figure (a). Note that the spectra have been staggered and the scale on the $y$-axis therefore is repeated.

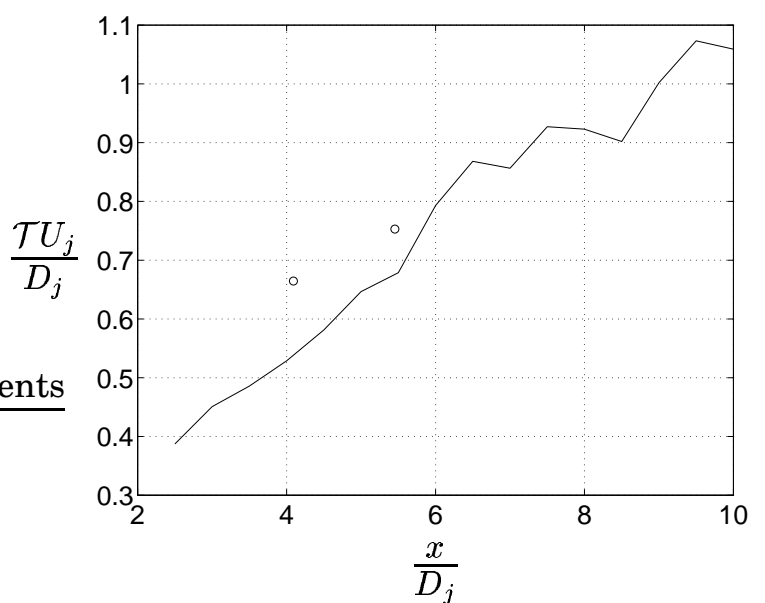

Fig. 26 Axial development of integral time scale in the shear layer, i.e. $r=0.5 D_{j}$. For legend see figure 25 . are very close to the Kirchhoff surface might effect the levels obtained. In figure 29 power spectrum of predicted pressure fluctuations in a couple of observer locations are compared with corresponding spectra obtained in the experiments. ${ }^{3}$ For low and high angles the predicted levels are in good agreement with the experimental data up to at least Strouhal number $S t=1.5$, see figure 29(a). For the intermediate angles on the other hand, the predicted amplitude decreases rapidly above $S t=1$. This is due to that the grid is not fine enough to support high frequency acoustic waves in the radial direction. Assuming that it is sufficient to have four cells per wavelength to be able to capture a propagating wave of a certain frequency, the cells in the area where the Kirchhoff surface is located would support propagating waves of Strouhal numbers up to $S t \simeq 1.2$.

In figures 30-31, autocorrelations of far-field pressure fluctuations are shown. Each figure contains autocorrelation curves for observer points at 


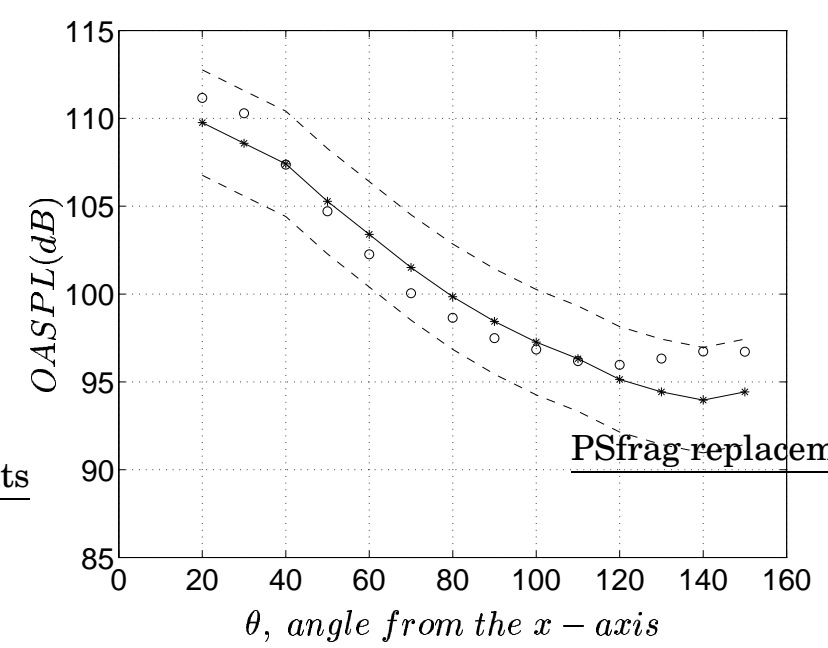

Fig. 27 Far-field Sound Pressure Levels in microphone locations $30 D_{j}$ from the nozzle exit: ' $-*$ ' corresponds to measured $^{3}$ sound pressure levels and the dashed lines, ' $--{ }^{\prime}$ to $3 \mathrm{~dB}$ measurement uncertainty. ' $O$ ' denotes calculated sound pressure levels using Kirchhoff surface integration.

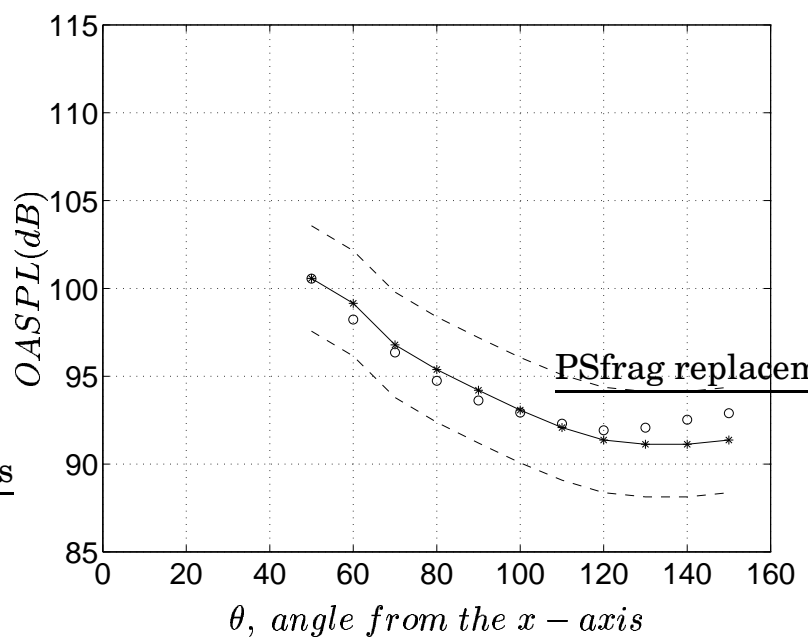

Fig. 28 Far-field Sound Pressure Levels in microphone locations $50 D_{j}$ from the nozzle exit. See also legend in figure 27.

four different angles from the $x$-axis. The autocorrelation of pressure fluctuations in an observer location, $\mathbf{x}$, is calculated as

$$
R_{p p}(\mathbf{x}, \tau)=\frac{\left\langle p^{\prime}(\mathbf{x}, t) p^{\prime}(\mathbf{x}, t+\tau)\right\rangle_{t}}{\left\langle p^{\prime 2}(\mathbf{x})\right\rangle_{t}}
$$

The correlations in figure 30 are obtained at a $30 D_{j}$ radius and those in figure 31 at $50 D_{j}$. The time separation, $\tau$, to the first minimum is significantly shorter for the autocorrelation curve obtained in the observer location 140 degrees from the $x$-axis, which indicates that more energy is radiated at higher frequencies at high angles.

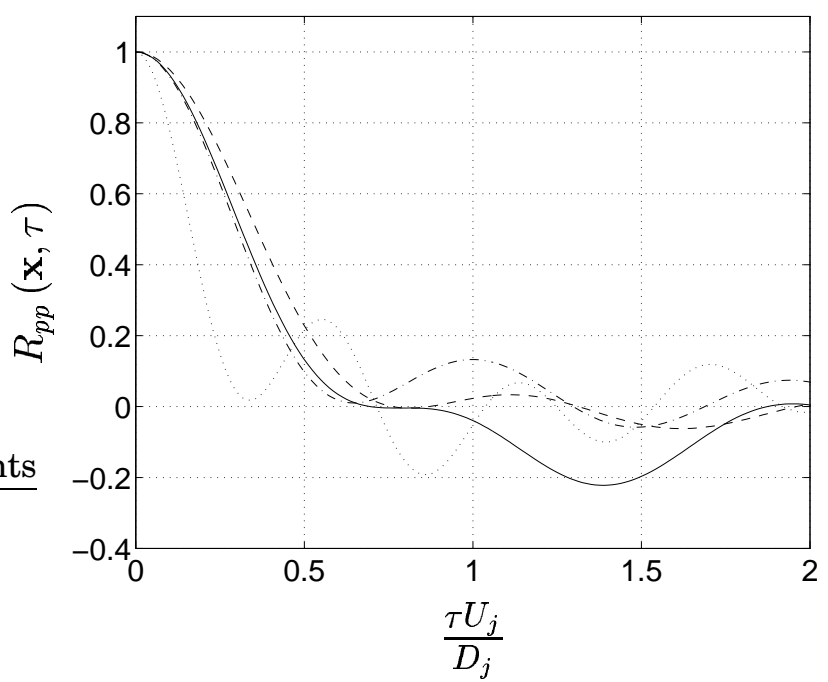

Fig. 30 Autocorrelation of far-field pressure fluctuations for observer locations, $\mathbf{x}$, on a $30 D_{j}$ radius circle. The solid line denotes a location 50 degrees from the $x$-axis, the dashed line, , $--{ }^{\prime}, 80$ degrees, $' \ldots{ }^{\prime} 110$ degrees and ${ }^{\prime} .{ }^{\prime} 140$ degrees.

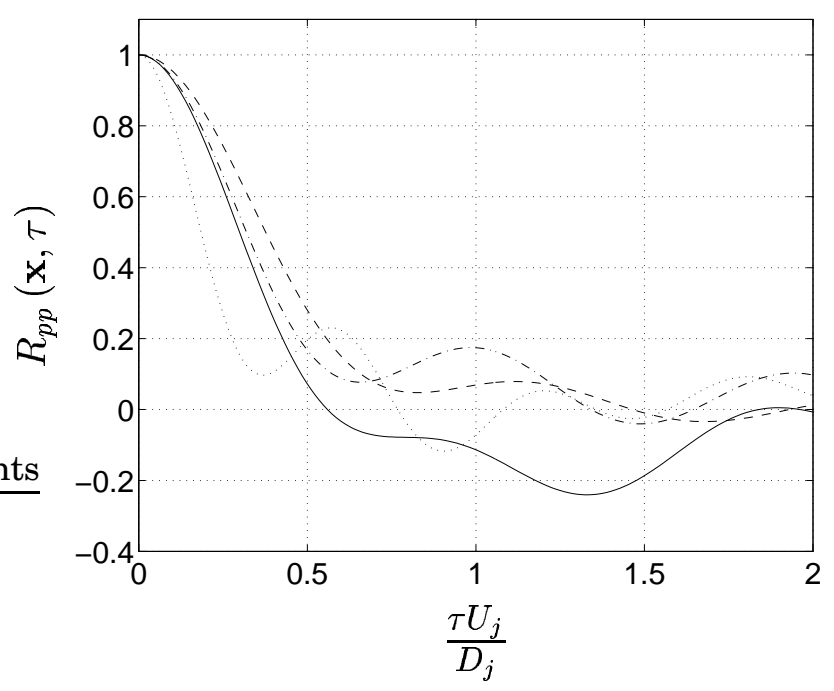

Fig. 31 Autocorrelation of far-field pressure fluctuations for observer locations, $\mathbf{x}$, on a $50 D_{j}$ radius circle. For legend see figure 30 .

\section{Computational resources}

The computations have been performed partly on a Sun-cluster using 14 SunBlade $750 \mathrm{MHz}$ processors and partly on a Linux-cluster using 14 AMD $1700^{+}$processors. MPI $^{3}$ routines have been implemented for communication between the processors.

\section{Concluding remarks}

An LES of a compressible Mach 0.75 nozzle/jet configuration has been performed. The Reynolds number based on jet velocity and nozzle diameter was 50 000. Profiles of time and azimuthally averaged flow properties have been compared with experimental results. ${ }^{1}$ Although some deviations occur, the results are in good agreement with exper-

\footnotetext{
${ }^{3}$ Message Passing Interface
} 
iments. The good results are probably attributed to the cartesian mesh used and the fact that a nozzle geometry has been included in the calculation domain. Using a cartesian mesh makes it possible to ensure that cells in the outer regions of the calculation domain are not too large and prevent cells from being clustered in the centerline region. The jet spreading and the potential core length are not predicted correctly. This might be due to use of too coarse grid in the nozzle outlet region, i.e. too coarse for the sub-grid scale model used. Furthermore, the entrainment velocities specified at the free boundaries probably affects the jet spreading.

Two-point space-time correlations have been obtained in a number of locations in the shear layer along the nozzle lip-line. Correlation curves obtained at $x=L_{c}$ were in good agreement with experiments. $^{2}$ Cross-correlations in the shear layer were used to obtain estimates of integral length scales, integral time scales and eddy convection velocities.

Sound pressure levels in the far-field have been evaluated using a hybrid approach. Kirchhoff surface integration was utilized for propagation of sound to far-field locations. Instantaneous pressure on the Kirchhoff surface was obtained from the LES. Sound pressure levels obtained are in excellent agreement with experimental results. ${ }^{3}$ However, although the results were in good agreement with experiments regarding sound pressure levels, the grid resolution was inadequate to be able to resolve acoustic waves of Strouhal numbers higher than $S t \simeq 1.2$. It is worth mentioning that these good results have been obtained although a less accurate numerical scheme than recommended for aeroacoustic applications have been used.

\section{Acknowledgment}

This work was conducted as part of the EU 5th Framework Project JEAN (Jet Exhaust Aerodynamics \& Noise), contract number G4RD-CT2000000313. Computer time at the Sun-cluster, provided by UNICC at Chalmers, is gratefully acknowledged.

\section{References}

${ }^{1}$ P. Jordan, Y. Gervais, J.-C. Valiére and H. Foulon, "Final results from single point measurements," Project deliverable D3.4, JEAN - EU 5th Framework Programme, G4RD-CT2000-00313, Laboratoire d'Etude Aeródynamiques, Poitiers, 2002.

${ }^{2}$ P. Jordan and Y. Gervais, "Modelling self and shear noise mechanisms in anisotropic turbulence," The 9th AIAA/CEAS Aeroacooustic Conference, AIAA 2003-8743, Hilton Head, South Carolina, 2003.

${ }^{3}$ P. Jordan, Y. Gervais, J.-C. Valiére and H. Foulon, "Results from acoustic field measurements," Project deliverable D3.6, JEAN - EU 5th Framework Programme, G4RD-CT2000-00313, Laboratoire d'Etude Aeródynamiques, Poitiers, 2002.

${ }^{4} \mathrm{~J}$. B. Freund, "Noise sources in a low-Reynolds-number turbulent jet at Mach 0.9," Journal of Fluid Mechanics, Vol. 438, 2001, pp. 277-305.
${ }^{5}$ B. E. Mitchell, S. K. Lele and P. Moin, "Direct computation of the sound generated by vortex pairing in an axisymetric jet," Journal of Fluid Mechanics, Vol. 383, 1999, pp. 113 - 142.

${ }^{6} \mathrm{C}$. Bogey, C. Bailly and D. Juvé, "Computation of the sound radiated by a 3-D jet using Large Eddy Simulation," The 6th AIAA/CEAS Aeroacooustic Conference, AIAA 2000-2009, Lahaina, Hawaii, 2000.

${ }^{7}$ R. R. Mankbadi, S. H. Shih, R. Hixon and L. A. Povinelli, "Direct Computation of Jet Noise Produced by Large-Scale Axisymmetric Structures," J. Propulsion and Power, Vol. 16 , No. 2, 2000, pp. 207-215.

${ }^{8}$ M. Billson, L.-E. Eriksson and L. Davidson, "Acoustic Source Terms for the Linear Euler Equations on Conservative form," The 8th AIAA/CEAS Aeroacoustics Conference, AIAA 2002-2582, Breckenridge, Colorado, 2002.

${ }^{9} \mathrm{C}$. Bogey, C. Bailly and D. Juvé, "Noise computation using Lighthill's equation with inclusion of mean flow - acoustic interactions," The 7th AIAA/CEAS Aeroacooustic Conference, AIAA 2001-2255, Maastricht, Netherlands, 2001.

${ }^{10}$ J. B. Freund, S. K. Lele and P. Moin, "Calculation of the Radiated Sound Field Using an Open Kirchhoff Surface," AIAA Journal, Vol. 34 , No. 5, 1996, pp. 909-916.

${ }^{11}$ R. R. Mankbadi, "Review of Computational Aeroacoustics in Propulsion Systems," J. Propulsion and Power, Vol. 15 , No. 4, 1999, pp. 504-512.

${ }^{12}$ M. Billson, "Computational Techniques for Jet Noise Predictions," Lic. Thesis, Department of Thermo and Fluid Dynamics, Chalmers University of Technology, Gothenburg, 2002.

${ }^{13}$ G. Erlebacher, M. Y. Hussaini, C. G. Speziale and T. A. Zang, "Toward the large-eddy simulation of compressible turbulent flows," Vol. ICASE-87-20, NASA-CR-178273.

${ }^{14}$ A. S. Lyrintzis, "Review: The use of Kirchhoff's method in computational aeroacoustics," ASME: Journal of Fluids Engineering, Vol. 116, 1994, pp. $665-676$.

${ }^{15}$ L.-E. Eriksson, "Development and validation of highly modular flow solver versions in G2DFLOW and G3DFLOW," Internal report 9970-1162, Volvo Aero Corporation, Sweden, 1995.

${ }^{16} \mathrm{C}$. Bogey, C. Bailly and D. Juvé, "Numerical simulation of sound generated by vortex pairing in a mixing layer," AIAA Journal, Vol. 38 , No. 12, 2000, pp. 2210-2218.

${ }^{17}$ T. Colonius, S. K. Lele and P. Moin, "Boundary conditions for direct computation of aerodynamic sound generation," AIAA Journal, Vol. 31 , No. 9, 1993, pp. 1574-1582.

${ }^{18}$ L.-E. Eriksson, "RANS prediction for test cases," Project deliverable D1.5, JEAN - EU 5th Framework Programme, G4RDCT2000-00313, Volvo Aero Corporation, Sweden, 2002.

${ }^{19}$ J. R. DeBonis, J. N. Scott, "A Large-Eddy Simulation of a Turbulent Compressible Round Jet," The 7th AIAA/CEAS Aeroacooustic Conference, AIAA 2001-2254, Maastricht, Netherlands, 2001.

${ }^{20}$ P. J. Morris, T. E. Scheidegger and L. N. Long, "Jet Noise Simulations for Circular Nozzles," The 6th AIAA/CEAS Aeroacooustic Conference, AIAA 2000-2009, Lahaina, Hawaii, 2000.

${ }^{21}$ H. J. Hussein, S. P. Capp and W. K. George, "Velocity measurements in a high-Reynolds-number, momentumconserving, axisymetric, turbulent jet," Journal of Fluid Mechanics, Vol. 258, 1994, pp. 31-75.

${ }^{22}$ M. J. Fisher and P. O. A. L. Davies, "Correlation measurements in a non-frozen pattern of turbulence," Journal of Fluid Mechanics, Vol. 18, 1963, pp. 97-116. 